\title{
Therapeutic Approach to Neurodegenerative Diseases by Medical Gases: Focusing on Redox Signaling and Related Antioxidant Enzymes
}

\author{
Kyota Fujita, ${ }^{1}$ Megumi Yamafuji, ${ }^{1}$ Yusaku Nakabeppu, ${ }^{2,3}$ and Mami Noda $^{1,3}$ \\ ${ }^{1}$ Laboratory of Pathophysiology, Graduate School of Pharmaceutical Sciences, Kyushu University, 3-1-1 Maidashi, Fukuoka 812-8582, \\ Japan \\ ${ }^{2}$ Division of Neurofunctional Genomics, Medical Institute of Bioregulation, Kyushu University, 3-1-1 Maidashi, Fukuoka 812-8582, \\ Japan \\ ${ }^{3}$ Research Center for Nucleotide Pool, Kyushu University, 3-1-1 Maidashi, Fukuoka 812-8582, Japan
}

Correspondence should be addressed to Mami Noda, noda@phar.kyushu-u.ac.jp

Received 2 March 2012; Accepted 25 April 2012

Academic Editor: Guilherme Antonio Behr

Copyright (c) 2012 Kyota Fujita et al. This is an open access article distributed under the Creative Commons Attribution License, which permits unrestricted use, distribution, and reproduction in any medium, provided the original work is properly cited.

Oxidative stress in the central nervous system is strongly associated with neuronal cell death in the pathogenesis of several neurodegenerative diseases such as Alzheimer's disease, Parkinson's disease, Huntington's disease, and amyotrophic lateral sclerosis. In order to overcome the oxidative damage, there are some protective signaling pathways related to transcriptional upregulation of antioxidant enzymes, such as heme oxygenase-1 (HO-1) and superoxide dismutase (SOD)-1/-2. Their expression is regulated by several transcription factors and/or cofactors like nuclear factor-erythroid 2 (NF-E2) related factor 2 (Nrf2) and peroxisome proliferator-activated receptor- $\gamma$ coactivator $1 \alpha$ (PGC- $1 \alpha)$. These antioxidant enzymes are associated with, and in some cases, prevent neuronal death in animal models of neurodegenerative diseases. They are activated by endogenous mediators and phytochemicals, and also by several gases such as carbon monoxide $(\mathrm{CO})$, hydrogen sulphide $\left(\mathrm{H}_{2} \mathrm{~S}\right)$, and hydrogen $\left(\mathrm{H}_{2}\right)$. These might thereby protect the brain from severe oxidative damage and resultant neurodegenerative diseases. In this paper, we discuss how the expression levels of these antioxidant enzymes are regulated. We also introduce recent advances in the therapeutic uses of medical gases against neurodegenerative diseases.

\section{Introduction}

The brain consumes 20 to $50 \%$ of total body oxygen $\left(\mathrm{O}_{2}\right)$ consumption, although it only accounts for $2 \%$ of the body weight, meaning that brain function is largely dependent on constitutive supply of $\mathrm{O}_{2}[1]$. Compared to the normal physiological condition, in which 2 to $5 \%$ of total oxygen consumed by cells is converted into reactive oxygen species (ROS) as a byproduct of mitochondrial respiration, excessive and unregulated production of ROS can occur in pathological conditions $[2,3]$. Therefore, scavenging and regulating the amount of ROS in the brain is important to maintain normal brain activity.

Although aberrant production of ROS in the central nervous system (CNS) is critically linked to several neurodegenerative diseases such as Alzheimer's disease (AD),
Parkinson's disease (PD), Huntington's disease (HD), and amyotrophic lateral sclerosis (ALS), a set of antioxidant defense system can save the brain from severe injuries [4-8]. Oxidative stress activates a stress response, and adaptation against ROS-derived cellular injury maintains the redox balance and protects cells from lethal damage [9]. This adaptive response often requires upregulation of endogenous antioxidant enzymes, and their expression levels can be regulated by several transcription factors. To date, the importance of transcriptional regulation of antioxidant enzymes is recognized as a route to the discovery of neuroprotective strategies. In this paper, we highlight two major transcriptional regulation factors, nuclear factor-erythroid 2 (NF-E2) related factor 2 (Nrf2), and peroxisome proliferator-activated receptor- $\gamma$ coactivator $1 \alpha$ (PGC-1 $\alpha)$. Also, we focus on the role of heme oxygenase-1 (HOlinebreak and superoxide dismutase (SOD) 
in neurodegenerative diseases, because these are the key components of antioxidant mechanism Figure 1. Finally, we would like to introduce recent research on several gases such as $\mathrm{CO}, \mathrm{H}_{2}$, and $\mathrm{H}_{2} \mathrm{~S}$ (now called medical gases), suggesting a new therapeutic approach against oxidative damage and resultant neurodegenerative diseases, most notably PD.

\section{Nrf2: a Master of Redox Homeostasis}

Nuclear factor-erythroid 2 (NF-E2) related factor 2 (Nrf2) is an important transcription factor and is recognized as a major contributor to the upregulation of multiple antioxidant defense system in response to oxidative stress. Nrf2 belongs to the cap'n'collar (CNC) family of basic region-leucine zipper (bZip)-type transcription factors [10]. NF-E2 is a heterodimeric protein which contains a large p45 and small p18 subunit [11]. Cloning of its cDNA revealed that $\mathrm{p} 45$ contains a cap'n'collar-(CNC-) type bZip domain [12]. The p45 subunit utilizes its CNC-bZip domain to form a heterodimer with $\mathrm{p} 18$; the latter has been identified as MafK, one of the small musculoaponeurotic fibrosarcoma oncogene (Maf) transcription factors [12, 13]. The heterodimer binds to an NF-E2 motif; the small Maf protein $\mathrm{p} 18$ confers DNA-binding activity to $\mathrm{p} 45$, while $\mathrm{p} 45$ activates transcription via its transactivation domain [13, 14]. Nrf2 binds to the antioxidant-responsive element (ARE) or the electrophile-responsive element (EpRE) $[15,16]$. ARE has been detected in the promoter or upstream promoter regions of the genes encoding phase II antioxidant enzymes including glutathione S-transferase subunits (GST-Ya, GSTP, GST-M1/M3, etc.), glutamate-cysteine ligase catalytic (GCLC) and glutamate-cysteine ligase modifier (GCLM) subunits, the thioredoxin (TRX) and peroxiredoxin (PRX) families, and $\mathrm{NAD}(\mathrm{P}) \mathrm{H}$ :quinone oxidoreductase (NQO-1) [17-21]. Heme oxygenase-1 (HO-1) is also one of the Nrf2-ARE pathway-derived upregulated factors [22, 23], and its transcriptional upregulation is also mediated by some other transcription factors such as AP-1, CREB, and NF- $\kappa B$ [24]. In the CNS, genetic or pharmacological activation of Nrf2-ARE pathway can confer resistance against neurodegenerative disease insults such as $\mathrm{AD}, \mathrm{PD}, \mathrm{HD}$, and ALS. A lentiviral vector encoding human Nrf2 reduced spatial learning deficits in aged APP/PS1 mice, a mouse model of $\mathrm{AD}$ [25]. Compared to neurons, astrocytes have a much greater ability to increase Nrf2-ARE pathwayderived gene expressions, as shown by a study using cortical neuronal cultures obtained from ARE-human placental alkaline phosphatase (hPAP) reporter mice [26], grown under condition where the mixed cortical cultures consist of 30\% astrocytes and 70\% neurons. Genetic-overexpression of Nrf2 in astrocytes using the glial fibrillary acidic protein (GFAP) promoter (GFAP-Nrf2) has shown protective effects against several animal models of neurodegeneration, for example, motor neuron degeneration produced by expressing mutant human superoxide dismutase 1 in ALS model mice [27], and dopaminergic neuronal loss by a neurotoxin (1-methyl-4-phenyl-1,2,3,6-tetrahydropyridine, MPTP) in PD model mice [28]. Transplantation of astrocytes infected with adenovirus-Nrf2 protects striatal mediumspiny neuron degeneration by a mitochondrial complex II inhibitor (3-nitropropionic acid or malonate) in HD model mice [29].

HO-1, one of the antioxidant enzymes upregulated by Nrf2, is thought to be highly associated with AD pathology. In $\mathrm{AD}$ brain, $\mathrm{HO}-1$ is expressed both in neurons and in astrocytes; $86 \%$ of GFAP-positive astrocytes in AD hippocampus exhibit HO-1 immunoreactivity, whereas those in age-matched normal tissues are in the range of only 6-7\% [30]. HO-1 overexpression in astrocyte by transient transfection of HMOX1 cDNA significantly decreased intracellular cholesterol concentrations and increased the level of at least four oxysterol species compared to untreated control cultures [30]. In mild cognitive impairment or early $\mathrm{AD}$, enhanced HO-1 expression stimulated astrocyte cholesterol biosynthesis, oxysterol formation, and cholesterol efflux (to sites of neuronal repair and for egress across the blood-brain barrier). Glial cholesterol efflux exceeds biosynthesis, and total cholesterol levels in affected brains are normal or diminished. Regulation of sterol homeostasis is important in $\mathrm{AD}$ pathology because a massive increase in the free cholesterol pool saturates the sterol efflux mechanism, which results in an increase in brain cholesterol levels and exacerbates amyloid deposition and neurodegeneration in advanced $\mathrm{AD}$. Upregulation of HO-1 has another therapeutic potential for clearance of tau protein by the ubiquitin-proteasome system (UPS) [30]. Proteasome activity is reduced in AD brain and amyloid beta (A $\beta)$ inhibits the UPS in cultured cells [31, 32]. This influence on UPS, for which heme-derived iron and $\mathrm{CO}$ are responsible, promotes intracellular degradation of $\alpha$ synuclein as observed in HMOX1-transfected M17 cells [33]. Therefore, HO-1 is highly associated with the therapeutic approach not only by its antioxidant function but also by its influence on proteasomal degradation of tau and $\alpha$ synuclein.

\section{The Role of PGC-1 $\alpha$ in Neurodegenerative Diseases}

Since its discovery over a decade ago, peroxisome proliferator-activated receptor- $\gamma$ coactivator $1 \alpha$ (PGC- $1 \alpha)$ has been implicated in energy homeostasis, adaptive thermogenesis, $\beta$-oxidation of fatty acids, and glucose metabolism [34]. The activity of PGC- $1 \alpha$ depends on its ability to form heteromeric complexes with a variety of transcription factors including nuclear respiratory factor 1 and 2 (NRF1 and NRF-2), and the nuclear hormone receptors [35]. In particular, NRF-1 and NRF-2 are transcriptional regulators that act on nuclear genes encoding for constituent subunits of the oxidative phosphorylation system including cytochrome c, complex I-V, and mitochondrial transcription factor A (Tfam) [36]. Tfam, a transcription factor that acts on the promoters within the D-loop region of mitochondrial DNA and regulates the replication and transcription of the mitochondrial genome, contains consensus-binding sites for both NRF-1 and NRF-2 [37].

Mice lacking PGC- $1 \alpha$ show a profound spongiform pattern of lesions in the striatum together with hyperactivity, 


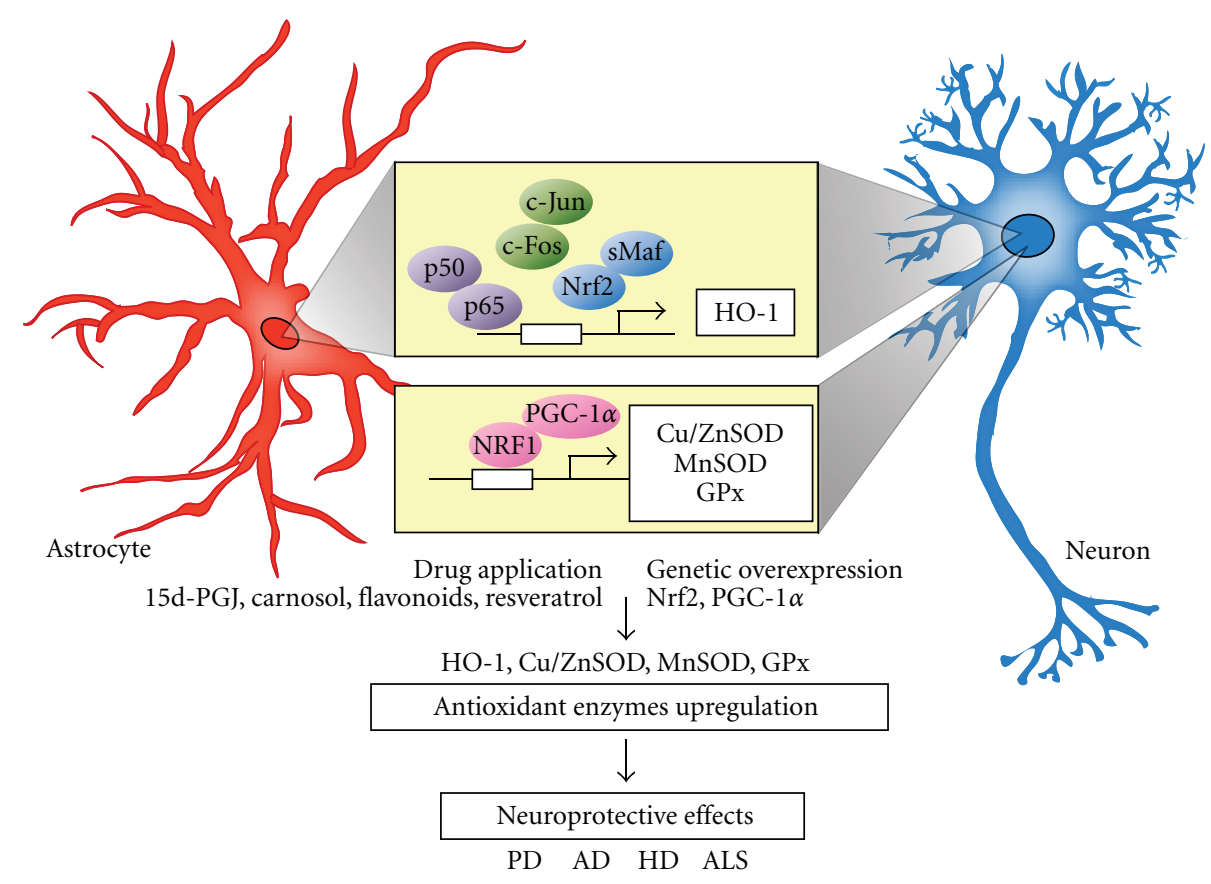

FIGURE 1: The transcriptional upregulation of antioxidant enzymes in neurodegenerative diseases. Both neurons and astrocytes can increase several antioxidant enzymes including heme oxygenase-1 (HO-1), copper and zinc-containing SOD (Cu/ZnSOD), manganesecontaining SOD (MnSOD), and glutathione peroxidase (GPx). By drug application or genetic overexpression of transcription factor, the transcriptional responses via NF- $\kappa \mathrm{B}$ (p50/p65), AP-1 (c-Jun/c-Fos), Nrf2/sMaf, and NRF1/PGC-1 $\alpha$ in response to oxidative stress and related neurodegenerative disease are activated.

which are the features of human HD [38]. In response to hydrogen peroxide $\left(\mathrm{H}_{2} \mathrm{O}_{2}\right)$, there is over a 6 -fold increase in PGC- $1 \alpha$ expression in mouse embryonic fibroblasts, as well as an increase of the transcription of mRNA encoding ROS defense enzymes such as copper/zinc superoxide dismutase (SOD1), manganese SOD (SOD2), catalase, and glutathione peroxidase (GPx) in association with the transcription factor, cAMP-responsive element binding protein (CREB). PGC- $1 \alpha$ expression is reduced by overexpression of mutant Huntingtin through its interference with formation of the CREB/TAF4 complex [39]. The HD striatal cell line, STHdhQ111 also shows reduced expression of PGC1$\alpha$ target genes encoding mitochondrial cytochrome $\mathrm{c}$ and cytochrome oxidase IV. On the other hand, lentiviral overexpression of mitogen- and stress-activated protein kinase1 increased PGC- $1 \alpha$ and protected against striatal lentiviral expression of polyglutamine expansion in huntingtin protein (Exp-Htt) [40]. Moreover, in a postmortem brain tissue study of HD patients, expression levels of 24 out of 26 PGC- $1 \alpha$ target genes were reduced, which implies that targeting PGC- $1 \alpha$ would be beneficial as a therapeutic approach for HD. PGC- $1 \alpha$ might also be beneficial for other neurodegenerative diseases such as PD, as reported in MPTPinduced PD model animals. PGC- $1 \alpha$-deficient mice were more sensitive to neurotoxic insult by MPTP [38], whereas overexpression of PGC- $1 \alpha$ protected neurons against MPTP neurotoxicity [41].

PGC- $1 \alpha$ activity is regulated by posttranslational modification including direct phosphorylation or deacetylation, which increases PGC- $1 \alpha$ activity or expression [42-46]. One such protein is NAD-dependent deacetylase Sir2. Its mammalian or human homologue, SIRT1, has been focused on as a prospective candidate for neuroprotective strategies against AD, PD, HD, and ALS [47-51]. One of the important roles of SIRT1 lies in its deacetylase activity, and its deacetylase substrates such as PGC- $1 \alpha$ and forkhead box $\mathrm{O} 3 \mathrm{~A}$ (Foxo3a) are involved in antioxidant responses and gene transcription [52]. Most especially, overexpression of SIRT1 deacetylase and suppression of GCN5 acetylase increase the transcriptional activity of PGC- $1 \alpha$ and prevent mitochondrial loss in neurons induced by expanded Huntingtin protein [53]. A recent study by Martin et al. has revealed that mitogen- and stress-activated kinase (MSK-1), a nuclear protein kinase involved in chromatin remodeling through histone $\mathrm{H} 3$ phosphorylation, is linked to the nucleosomal response at the PGC- $1 \alpha$ promoter, and transcription via CREB phosphorylation [40].

Among the genes regulated by NRF-1 and/or PGC- $1 \alpha$, SOD1 and 2 are dominant and the first lines of defense against ROS, especially the superoxide anion radical $\left(\mathrm{O}_{2}{ }^{-{ }^{-}}\right)$, are catalyzed to molecular oxygen and hydrogen peroxide [54]. In humans, three different forms of SOD are reported: SOD1, SOD2 in mitochondria, and extracellular $\mathrm{Cu} / \mathrm{ZnSOD}$ (SOD3) [55-57]. SOD1 and SOD2 are abundant in the CNS, whereas SOD3 is less abundant than SOD1 and SOD2 [58]. The expression levels of SOD1 and SOD2 are associated with human amyloid precursor protein (hAPP)-/A $\beta$-induced impairments in aged mouse brain. SOD1 overexpression 
protects against the in vitro neurotoxicity induced by $\mathrm{A} \beta$ [59]. In vivo, coexpression of hSOD1 with an APP transgene protects against the lethal effects of APP [60]. SOD2 is enriched around amyloid plaques $[61,62]$ and brain microvessels [63] in hAPP transgenic mice but decreased in $\mathrm{AD}$ brains overall [64]. Esposito et al. has shown that partial reduction in the main mitochondrial superoxide scavenger SOD2 using SOD2 ${ }^{+/-}$mice accelerates the onset of hAPP/A $\beta$-dependent behavioral abnormalities and worsens a range of $\mathrm{AD}$-related molecular and pathological alterations [65]. On the other hand, overexpression of SOD2 reduces hippocampal superoxide and prevents memory deficits in the Tg2576 mouse model of AD that overexpresses the hAPP carrying the Swedish mutation (K670N:M671L) [66].

\section{Reducing ROS by Medical Gases}

The generation of ROS and related oxidative damage are believed to be involved in the pathogenesis of neurodegenerative diseases. The main ROS involved in the pathogenesis of neurodegeneration are $\mathrm{O}_{2}{ }^{\bullet-}, \mathrm{H}_{2} \mathrm{O}_{2}$, and the highly reactive hydroxyl radical $\left(\mathrm{HO}^{\bullet}\right)$. Recently, there have been increasing reports showing that medical gases, such as carbon monoxide (CO), nitric oxide ( $\mathrm{NO})$, and hydrogen sulfide $\left(\mathrm{H}_{2} \mathrm{~S}\right)$ as well as molecular hydrogen $\left(\mathrm{H}_{2}\right)$, might overcome the harmful damage produced by oxidative stress $[67,68]$. These gases directly eliminate ROS, or induce resistant proteins and antioxidant enzymes to antagonize oxidative stress Table 1.

4.1. Carbon Monoxide. CO is a diatomic molecule and is soluble in aqueous media and organic solvents [69]. Not only exogenous environmental exposure but also endogenous production during heme metabolism are major sources of CO from primitive prokaryocytes to human $[69,70]$. Endogenous production of $\mathrm{CO}$ is highly associated with $\mathrm{HO}-$ 1 activity which induces enzymatic degradation of heme. $\mathrm{HO}$ breaks the alpha-methylene carbon bond of the porphyrin ring using $\mathrm{NADPH}$ and molecular $\mathrm{O}_{2}$ in a reaction that releases equimolar amount of biliverdin, iron, and CO [71, 72].

Recent studies have revealed that $\mathrm{CO}$ serves as an intrinsic signaling molecule and shows anti-inflammatory and antiapoptotic effects. These effects of $\mathrm{CO}$ are mediated by 338 mitogen-activated protein kinase (MAPK) signaling, which is activated in response to physical and chemical stress inducers including oxidative stress, UV light, ischemia, and proinflammatory cytokines [73]. Activation of p38 also mediates the induction of heat shock protein (Hsp72) via its transcriptional factor, heat shock factor-1, leading to the cytoprotective effects [74].

Exogenous $\mathrm{CO}$ also activates Nrf2 pathway and decreased infarct size in an ischemia/reperfusion model [75]. Nrf2 activation can coordinately upregulate expression of several antioxidative enzymes recognized to play important roles in combating oxidative stress, including HO-1. Endogenous $\mathrm{CO}$, which is produced by heme degradation, induces ROSdependent signal transduction in the mitochondrial SOD2 and in HO-1 itself [76].
4.2. Hydrogen Sulfide. $\mathrm{H}_{2} \mathrm{~S}$ is a flammable, water-soluble gas with a smell of rotten eggs and is known as a toxic gas and as an environmental hazard. The production of $\mathrm{H}_{2} \mathrm{~S}$ from Lcysteine is catalysed primarily by two enzymes, cystathionine $\gamma$-lyase (CSE) and cystathionine $\beta$-synthase (CBS). Although exposure to higher levels $(\sim \mathrm{mM})$ of $\mathrm{H}_{2} \mathrm{~S}$ is cytotoxic (free radical generation, glutathione depletion, intracellular iron increase, and mitochondrial cell death signal), lower concentration $(\sim \mu \mathrm{M})$ of $\mathrm{H}_{2} \mathrm{~S}$ shows cytoprotective (antinecrotic and antiapoptotic) effects.

Biochemical analysis has revealed that sulphide shows a direct antioxidant reaction with one- or two-electron molecules (one-electron molecules: $\bullet \mathrm{NO}_{2}, \bullet \mathrm{OH}, \mathrm{CO}_{3}^{\bullet-}$, two-electron molecules: peroxynitrite, hydrogen peroxide, hypochlorite, taurine, and chloramine) as well as other low-molecular-weight thiol molecules such as cysteine and glutathione. Although sulphide is not a preferential target for radicals or oxidants due to its low concentration in vivo, it can serve as a direct antioxidant [77, 78]. $\mathrm{H}_{2} \mathrm{~S}$ can also induce upregulation of transcription for antiinflammatory and cytoprotective genes including HO-1 [79, 80]. By upregulating $\mathrm{HO}-1$ expression, $\mathrm{H}_{2} \mathrm{~S}$ can trigger the production of $\mathrm{CO}$, which shows anti-inflammatory and antiapoptotic effects.

4.3. Hydrogen. Since the first striking evidence indicating that molecular hydrogen acts as an antioxidant and inhalation of hydrogen-containing gas reduces ischemic injury in brain [81], there have been increasing numbers of reports which support the therapeutic properties of hydrogen against oxidative stress-related diseases and damages in brain [82, 83], liver [84], intestinal graft [85], myocardial injury [86, 87], and atherosclerosis [88]. Hydrogen can be taken up by inhalation of hydrogen-containing air (hydrogen gas) or drinking hydrogen-containing water (hydrogen water). One hour after the start of inhalation of hydrogen gas, hydrogen can be detected in blood, at levels of $10 \mu \mathrm{M}$ in arterial blood [81]. The content of hydrogen can be measured even after intake of hydrogen water by a catheter, which yields $5 \mu \mathrm{M}$ in arterial blood calculated after $3 \mathrm{~min}$ of hydrogen water incorporation [82]. Taking into account its continuous intake, it is easier and safer to drink hydrogen water than inhaling hydrogen gas.

We have previously showed that $\mathrm{H}_{2}$ in drinking water reduced the loss of dopaminergic neurons in MPTP-induced Parkinson's disease (PD) mice [89]. The therapeutic effects of $\mathrm{H}_{2}$ water were observed in another PD model, 6-OHDAtreated rats [90]. In these animal models, administration of neurotoxins decreased the number of dopaminergic neurons in the substantia nigra pars compacta (SNpc), as well as dopaminergic nerve terminal fibers in the striatum. However, taking $\mathrm{H}_{2}$ water significantly reduced the loss of both neuronal cell bodies and fibers compared with the controls drinking normal water. Mice chronically treated with MPTP using an osmotic pump also showed behavioral impairments observed by open-field test [91], and rats administered with 6-OHDA showed behavioral impairments assessed by the rotarod test. Hydrogen improved these 
TABLE 1: Reducing ROS by medical gases, hydrogen $\left(\mathrm{H}_{2}\right)$, carbon monoxide $(\mathrm{CO})$, and hydrogen sulphide $\left(\mathrm{H}_{2} \mathrm{~S}\right)$. These gases can increase endogenous antioxidant enzymes and stress response protein such as HO-1, SOD, and Hsp72. Hydrogen and hydrogen sulphide can directly react with ROS and show radical scavenging effects.

\begin{tabular}{|c|c|c|c|c|}
\hline Medical gases & Direct reaction to ROS & Reference & $\begin{array}{c}\text { Endogenous cytoprotective protein } \\
\text { induction }\end{array}$ & Reference \\
\hline \multirow{2}{*}{ Hydrogen $\left(\mathrm{H}_{2}\right)$} & \multirow{2}{*}{$\begin{array}{c}\text { Radical scavenging } \\
\mathrm{HO}^{\bullet}+\mathrm{H}_{2} \longrightarrow \mathrm{H}_{2} \mathrm{O}+\mathrm{H}^{\bullet}\end{array}$} & \multirow[t]{2}{*}[67]{} & Increase superoxide dismutase (SOD) & {$[81]$} \\
\hline & & & $\begin{aligned} & \text { Increase bilirubin } \\
& \fallingdotseq \text { induction of } \mathrm{HO}-1 \\
&\end{aligned}$ & {$[81]$} \\
\hline \multirow{3}{*}{ Carbon monoxide (CO) } & & & $\begin{array}{l}\text { Activation of p38 MAPK signaling } \\
\text { p38 } \longrightarrow \mathrm{HSF} 1 \longrightarrow \mathrm{Hsp72}\end{array}$ & {$[59]$} \\
\hline & & & $\begin{array}{l}\text { Activation of Nrf2 pathway } \\
\qquad \mathrm{Nrf}-2 \longrightarrow \mathrm{HO}-1\end{array}$ & {$[61]$} \\
\hline & & & $\begin{array}{l}\text { Induce superoxide dismutase (SOD2) } \\
\text { and } \mathrm{HO}-1\end{array}$ & {$[62]$} \\
\hline \multirow[t]{2}{*}{ Hydrogen sulphide $\left(\mathrm{H}_{2} \mathrm{~S}\right)$} & Radical scavenging & {$[64]$} & $\begin{array}{l}\text { Upregulation of cytoprotective genes } \\
\text { including HO-1 }\end{array}$ & {$[65]$} \\
\hline & $2 \mathrm{O}_{2}^{\cdot-}+\mathrm{H}_{2} \mathrm{~S} \longrightarrow \mathrm{HS}-\mathrm{SH}+\mathrm{O}_{2}+2 \mathrm{OH}^{-}$ & {$[63]$} & & {$[66]$} \\
\hline
\end{tabular}

behavioral impairments in both of these animal models of PD.

In the first report [81], $\mathrm{H}_{2}$ selectively reduced cytotoxic - $\mathrm{OH}$ radicals, whereas the production of other radicals such as superoxide, hydrogen peroxide, and nitric oxide was not altered. This selectivity was verified in a cell-free system, and in particular, the preference for scavenging $\bullet \mathrm{OH}$ rather than superoxide was confirmed in PC12 cell culture system. According to Setsukinai et al. [92], both $\bullet \mathrm{OH}$ and peroxynitrite $\left(\mathrm{ONOO}^{-}\right)$are much more reactive than other ROS. This would explain why $\mathrm{H}_{2}$ shows a selective reaction with only the strongest radicals, both in the cell-free system and in PC12 cells.

Especially, $\bullet \mathrm{OH}$ overproduction in oxidative and neurotoxic reaction by MPTP leads to lipid peroxidation in nigral dopaminergic neurons prior to cell death, observed by 4hydroxynonenal (4-HNE) immunostaining, the markers of membrane lipid peroxidation. Immunoreactivity of 4-HNE in MPTP-treated mice is increased by three times compared to that in saline-treated mice [89], which is similar to the previous report of 4-HNE protein levels in substantia nigra observed at the same time after MPTP administration using HPLC [93]. $\mathrm{H}_{2}$ water significantly reduces the formation of 4-HNE in dopaminergic neurons in substantia nigra to the level of control [89]. MPTP-induced loss of dopaminergic neuron is associated with the accumulation of 8-oxo-7, 8dihydroguanine (8-oxoG) in the dopaminergic neurons. $\mathrm{H}_{2}$ water significantly decreased this accumulation in the striatum [89]. 8-oxoG is the major oxidized form of guanine in DNA, RNA and nucleotide pool by $\bullet \mathrm{OH}$ and is accumulated in both mitochondrial and nuclear DNA; their nomenclature are mt8oxo-G and nu8-oxoG, respectively. Mt8oxoG accumulates in the striatum which is rich in mitochondria in nerve terminals of dopaminergic neurons projecting from the substantia nigra. Although nu8oxoG was not detected in the nigral cell nucleus, $\mathrm{H}_{2}$ water might prevent the mt8oxoGinduced cellular apoptotic signals, not just reduce $\bullet \mathrm{OH}$ in the dopaminergic nerve terminal. On the other hand, the increase in $\mathrm{O}_{2}{ }^{\bullet-}$, which was detected by the $\mathrm{O}_{2}{ }^{--}$indicator, dihydroethidium (DHE), was not significantly decreased by $\mathrm{H}_{2}$ water [89]. Although $\mathrm{H}_{2}$ prevented superoxide formation in brain slices in hypoxia/reperfusion injury [94], $\mathrm{H}_{2}$ water might show a preferential reduction of $\bullet \mathrm{OH}$ during the protection of dopaminergic neurons.

Initial evidence suggests that $\mathrm{H}_{2}$ protects cells and tissues against strong oxidative stress by scavenging $\bullet \mathrm{OH}$ [81]. Also, $\mathrm{H}_{2}$ was effective when it was inhaled during reperfusion; when $\mathrm{H}_{2}$ was inhaled just during the initial ischemia (not in the reperfusion stage), infarct volume was not significantly decreased. It was shown that hydrogen in the brain decreased immediately after stopping inhalation and completely disappeared within $10 \mathrm{~min}$ [89], indicating that the effect of hydrogen can be observed only during the period when the oxidative insults occur. According to a previous report [82], $\mathrm{H}_{2}$ could be detected in the blood 3 minutes after administration of $\mathrm{H}_{2}$ water into the stomach. However, unpublished data showed that the half-life of $\mathrm{H}_{2}$ in the muscle in rats was approximately 20 minutes after the administration of $\mathrm{H}_{2}$ gas. Taking these reports into consideration, $\mathrm{H}_{2}$ in the brain and other tissues does not stay long enough to exert its ability as an antioxidant to ROS directly. Therefore, it is unlikely that direct reaction of $\mathrm{H}_{2}$ itself with ROS plays a major role in the neuroprotection, especially with $\mathrm{H}_{2}$ in drinking water, even though $\mathrm{H}_{2}$ itself has the ability to reduce $\bullet \mathrm{OH}$ preferentially. In accordance with this hypothesis, previous reports from Nakao et al. has demonstrated that drinking hydrogen water increased the amount of antioxidant enzyme, superoxide dismutase (SOD) [95], an endogenous defensive system against ROSinduced cellular damage. It was also reported that $\mathrm{H}_{2}$-water increases total bilirubin for four to eight weeks compared to baseline. Bilirubin is produced by the catalytic reaction of HO-1, and degradation of heme generates bilirubin as well as $\mathrm{CO}$ and free iron. Therefore, taking these observations into consideration, there seem to be other mechanisms for protective effect of $\mathrm{H}_{2}$ in drinking water, different from that exerted by $\mathrm{H}_{2}$ inhalation. It is possible that drinking $\mathrm{H}_{2}$ water has not only the ability to reduce cytotoxic radicals, 
but also brings into play novel mechanisms which are related to antioxidative defense system.

\section{Conclusion}

Recent advance in understanding of the regulation of antioxidant enzyme expression by transcriptional factors has given us the possibility that we can overcome several diseases mediated or induced by oxidative stress. As discussed above, transcriptional upregulation of several antioxidant enzymes like HO-1 and SOD might be beneficial for several neurodegenerative diseases such as AD, PD, HD, and ALS. Several phytochemicals (resveratrol, curcumin, flavonoids, carnosol, etc.) and endogenous mediators (15-deoxy- $\Delta^{12,14}$ PGJ2) can upregulate the antioxidants via transcription factors such as Nrf2, NF- $\kappa$ B, and AP-1 [24]. Surprisingly, HO1 and SOD are increased by $\mathrm{CO}, \mathrm{H}_{2}$ and $\mathrm{H}_{2} \mathrm{~S}$, although we cannot say whether these gases accelerate the stress response signaling or some transcriptional regulation system mediated by $\mathrm{Nrf} 2$ and PGC- $1 \alpha$. Together with the fact that $\mathrm{H}_{2}$, and $\mathrm{H}_{2} \mathrm{~S}$ themselves have the ability to react with ROS directly, we strongly suggest that these gases can buffer the ROS and in addition might prevent and/or protect the neurons from oxidative stress damages in neurodegenerative diseases.

\section{Acknowledgments}

The authors thank Professor D. A. Brown (University College London, UK) for valuable suggestion and reading the paper. This work was supported by Grants-in-Aid for Scientific Research of Japan Society for Promotion of Science.

\section{References}

[1] S. Papa, "Mitochondrial oxidative phosphorylation changes in the life span. Molecular aspects and physiopathological implications," Biochimica et Biophysica Acta, vol. 1276, no. 2, pp. 87-105, 1996.

[2] A. Boveris, N. Oshino, and B. Chance, "The cellular production of hydrogen peroxide," Biochemical Journal, vol. 128, no. 3, pp. 617-630, 1972.

[3] D. F. Stowe and A. K. S. Camara, "Mitochondrial reactive oxygen species production in excitable cells: modulators of mitochondrial and cell function," Antioxidants and Redox Signaling, vol. 11, no. 6, pp. 1373-1414, 2009.

[4] P. H. Reddy, "Mitochondrial medicine for aging and neurodegenerative diseases," NeuroMolecular Medicine, vol. 10, no. 4, pp. 291-315, 2008.

[5] R. H. Swerdlow and S. M. Khan, "A "mitochondrial cascade hypothesis" for sporadic Alzheimer's disease," Medical Hypotheses, vol. 63, no. 1, pp. 8-20, 2004.

[6] M. F. Beal, "Mitochondria take center stage in aging and neurodegeneration," Annals of Neurology, vol. 58, no. 4, pp. 495-505, 2005.

[7] D. C. Wallace, "A mitochondrial paradigm of metabolic and degenerative diseases, aging, and cancer: a dawn for evolutionary medicine," Annual Review of Genetics, vol. 39, pp. 359-407, 2005.
[8] E. Trushina and C. T. McMurray, "Oxidative stress and mitochondrial dysfunction in neurodegenerative diseases," Neuroscience, vol. 145, no. 4, pp. 1233-1248, 2007.

[9] Y. Miura and T. Endo, "Survival responses to oxidative stress and aging," Geriatrics and Gerontology International, vol. 10, supplement 1, pp. S1-S9, 2010.

[10] P. Moi, K. Chan, I. Asunis, A. Cao, and Y. W. Kan, "Isolation of NF-E2-related factor 2 (Nrf2), a NF-E2-like basic leucine zipper transcriptional activator that binds to the tandem NF-E2/AP1 repeat of the $\beta$-globin locus control region," Proceedings of the National Academy of Sciences of the United States of America, vol. 91, no. 21, pp. 9926-9930, 1994.

[11] P. A. Ney, N. C. Andrews, S. M. Jane et al., "Purification of the human NF-E2 complex: cDNA cloning of the hematopoietic cell-specific subunit and evidence for an associated partner," Molecular and Cellular Biology, vol. 13, no. 9, pp. 5604-5612, 1993.

[12] N. C. Andrews, H. Erdjument-Bromage, M. B. Davidson, P. Tempst, and S. H. Orkin, "Erythroid transcription factor NFE2 is a haematopoietic-specific basic-leucine zipper protein," Nature, vol. 362, no. 6422, pp. 722-728, 1993.

[13] K. Igarashi, K. Kataoka, K. Itoh, N. Hayashi, M. Nishizawa, and M. Yamamoto, "Regulation of transcription by dimerization of erythroid factor NF-E2 p45 with small Maf proteins," Nature, vol. 367, no. 6463, pp. 568-572, 1994.

[14] K. Igarashi, K. Itoh, H. Motohashi et al., "Activity and expression of murine small Maf family protein MafK," The Journal of Biological Chemistry, vol. 270, no. 13, pp. 76157624, 1995.

[15] T. H. Rushmore and C. B. Pickett, "Transcriptional regulation of the rat glutathione S-transferase Ya subunit gene. Characterization of a xenobiotic-responsive element controlling inducible expression by phenolic antioxidants," The Journal of Biological Chemistry, vol. 265, no. 24, pp. 14648-14653, 1990.

[16] R. S. Friling, A. Bensimon, Y. Tichauer, and V. Daniel, "Xenobiotic-inducible expression of murine glutathione Stransferase Ya subunit gene is controlled by an electrophileresponsive element," Proceedings of the National Academy of Sciences of the United States of America, vol. 87, no. 16, pp. 6258-6262, 1990.

[17] M. Kobayashi and M. Yamamoto, "Nrf2-Keap1 regulation of cellular defense mechanisms against electrophiles and reactive oxygen species," Advances in Enzyme Regulation, vol. 46, no. 1, pp. 113-140, 2006.

[18] J. A. Johnson, D. A. Johnson, A. D. Kraft et al., "The Nrf2ARE pathway: an indicator and modulator of oxidative stress in neurodegeneration," Annals of the New York Academy of Sciences, vol. 1147, pp. 61-69, 2008.

[19] N. G. Innamorato, A. I. Rojo, Á. J. García-Yagüe, M. Yamamoto, M. L. de Ceballos, and A. Cuadrado, "The transcription factor Nrf2 is a therapeutic target against brain inflammation," Journal of Immunology, vol. 181, no. 1, pp. 680-689, 2008.

[20] K. J. Hintze, A. S. Keck, J. W. Finley, and E. H. Jeffery, "Induction of hepatic thioredoxin reductase activity by sulforaphane, both in Hepalclc7 cells and in male Fisher 344 rats," Journal of Nutritional Biochemistry, vol. 14, no. 3, pp. 173-179, 2003.

[21] T. Ishii, K. Itoh, H. Sato, and S. Bannai, "Oxidative stressinducible proteins in macrophages," Free Radical Research, vol. 31, no. 4, pp. 351-355, 1999.

[22] L. V. Favreau and C. B. Pickett, "The rat quinone reductase antioxidant response element. Identification of the nucleotide sequence required for basal and inducible activity and detection of antioxidant response element-binding proteins 
in hepatoma and non-hepatoma cell lines," The Journal of Biological Chemistry, vol. 270, no. 41, pp. 24468-24474, 1995.

[23] T. Prestera, P. Talalay, J. Alam, Y. I. Ahn, P. J. Lee, and A. M. Choi, "Parallel induction of heme oxygenase- 1 and chemoprotective phase 2 enzymes by electrophiles and antioxidants: regulation by upstream antioxidant-responsive elements (ARE)," Molecular Medicine, vol. 1, no. 7, pp. 827-837, 1995.

[24] M. L. Ferrandiz and I. Devesa, "Inducers of heme oxygenase1," Current Pharmaceutical Design, vol. 14, no. 5, pp. 473-486, 2008.

[25] K. Kanninen, R. Heikkinen, T. Malm et al., "Intrahippocampal injection of a lentiviral vector expressing Nrf2 improves spatial learning in a mouse model of Alzheimer's disease," Proceedings of the National Academy of Sciences of the United States of America, vol. 106, no. 38, pp. 16505-16510, 2009.

[26] D. A. Johnson, G. K. Andrews, W. Xu, and J. A. Johnson, "Activation of the antioxidant response element in primary cortical neuronal cultures derived from transgenic reporter mice," Journal of Neurochemistry, vol. 81, no. 6, pp. 1233-1241, 2002.

[27] M. R. Vargas, D. A. Johnson, D. W. Sirkis, A. Messing, and J. A. Johnson, "Nrf2 activation in astrocytes protects against neurodegeneration in mouse models of familial amyotrophic lateral sclerosis," The Journal of Neuroscience, vol. 28, no. 50, pp. 13574-13581, 2008.

[28] P. C. Chen, M. R. Vargas, A. K. Pani et al., "Nrf2-mediated neuroprotection in the MPTP mouse model of Parkinson's disease: critical role for the astrocyte," Proceedings of the National Academy of Sciences of the United States of America, vol. 106, no. 8, pp. 2933-2938, 2009.

[29] M. J. Calkins, R. J. Jakel, D. A. Johnson, K. Chan, W. K. Yuen, and J. A. Johnson, "Protection from mitochondrial complex II inhibition in vitro and in vivo by Nrf2-mediated transcription," Proceedings of the National Academy of Sciences of the United States of America, vol. 102, no. 1, pp. 244-249, 2005.

[30] H. M. Schipper, W. Song, H. Zukor, J. R. Hascalovici, and D. Zeligman, "Heme oxygenase-1 and neurodegeneration: expanding frontiers of engagement," Journal of Neurochemistry, vol. 110, no. 2, pp. 469-485, 2009.

[31] A. Ciechanover and P. Brundin, "The ubiquitin proteasome system in neurodegenerative diseases: sometimes the chicken, sometimes the egg," Neuron, vol. 40, no. 2, pp. 427-446, 2003.

[32] B. S. Shastry, "Neurodegenerative disorders of protein aggregation," Neurochemistry International, vol. 43, no. 1, pp. 1-7, 2003.

[33] W. Song, A. Patel, H. Y. Qureshi, D. Han, H. M. Schipper, and H. K. Paudel, "The Parkinson disease-associated A30P mutation stabilizes $\alpha$-synuclein against proteasomal degradation triggered by heme oxygenase-1 over-expression in human neuroblastoma cells," Journal of Neurochemistry, vol. 110, no. 2, pp. 719-733, 2009.

[34] P. Puigserver and B. M. Spiegelman, "Peroxisome proliferatoractivated receptor $\gamma$ coactivator $1 \alpha$ (PGC- $1 \alpha)$ : transcriptional coactivator and metabolic regulator," Endocrine Reviews, vol. 24, no. 1, pp. 78-90, 2003.

[35] M. T. Lin and M. F. Beal, "Mitochondrial dysfunction and oxidative stress in neurodegenerative diseases," Nature, vol. 443, no. 7113, pp. 787-795, 2006.

[36] D. P. Kelly and R. C. Scarpulla, "Transcriptional regulatory circuits controlling mitochondrial biogenesis and function," Genes and Development, vol. 18, no. 4, pp. 357-368, 2004.
[37] R. C. Scarpulla, "Transcriptional paradigms in mammalian mitochondrial biogenesis and function," Physiological Reviews, vol. 88, no. 2, pp. 611-638, 2008.

[38] J. St-Pierre, S. Drori, M. Uldry et al., "Suppression of reactive oxygen species and neurodegeneration by the PGC-1 transcriptional coactivators," Cell, vol. 127, no. 2, pp. 397-408, 2006.

[39] L. Cui, H. Jeong, F. Borovecki, C. N. Parkhurst, N. Tanese, and D. Krainc, "Transcriptional repression of PGC- $1 \alpha$ by mutant huntingtin leads to mitochondrial dysfunction and neurodegeneration," Cell, vol. 127, no. 1, pp. 59-69, 2006.

[40] E. Martin, S. Betuing, C. Pagès et al., "Mitogen- and stressactivated protein kinase 1-induced neuroprotection in Huntington's disease: role on chromatin remodeling at the PGC-1alpha promoter," Human Molecular Genetics, vol. 20, no. 12, pp. 2422-2434, 2011.

[41] G. Mudo, J. Makela, V. Di Liberto et al., "Transgenic expression and activation of PGC-1alpha protect dopaminergic neurons in the MPTP mouse model of Parkinson's disease," Cellular and Molecular Life Sciences, vol. 69, no. 7, article 1153, 2012.

[42] M. Lagouge, C. Argmann, Z. Gerhart-Hines et al., "Resveratrol improves mitochondrial function and protects against metabolic disease by activating SIRT1 and PGC-1 $\alpha$," Cell, vol. 127, no. 6, pp. 1109-1122, 2006.

[43] H. Zong, J. M. Ren, L. H. Young et al., "AMP kinase is required for mitochondrial biogenesis in skeletal muscle in response to chronic energy deprivation," Proceedings of the National Academy of Sciences of the United States of America, vol. 99, no. 25, pp. 15983-15987, 2002.

[44] S. Jager, C. Handschin, J. St-Pierre, and B. M. Spiegelman, "AMP-activated protein kinase (AMPK) action in skeletal muscle via direct phosphorylation of PGC-1 $\alpha$," Proceedings of the National Academy of Sciences of the United States of America, vol. 104, no. 29, pp. 12017-12022, 2007.

[45] T. F. Outeiro, O. Marques, and A. Kazantsev, "Therapeutic role of sirtuins in neurodegenerative disease," Biochimica et Biophysica Acta, vol. 1782, no. 6, pp. 363-369, 2008.

[46] D. J. Ho, N. Y. Calingasan, E. Wille, M. Dumont, and M. F. Beal, "Resveratrol protects against peripheral deficits in a mouse model of Huntington's disease," Experimental Neurology, vol. 225, no. 1, pp. 74-84, 2010.

[47] J. Gao, W. Y. Wang, Y. W. Mao et al., "A novel pathway regulates memory and plasticity via SIRT1 and miR-134," Nature, vol. 466, no. 7310, pp. 1105-1109, 2010.

[48] G. Donmez, A. Arun, C. Y. Chung et al., "SIRT1 protects against $\alpha$-synuclein aggregation by activating molecular chaperones," The Journal of Neuroscience, vol. 32, no. 1, pp. 124$132,2012$.

[49] M. Jiang, J. Wang, J. Fu et al., "Neuroprotective role of Sirt1 in mammalian models of Huntington's disease through activation of multiple Sirt1 targets," Nature Medicine, vol. 18, pp. 153-158, 2012.

[50] D. Kim, M. D. Nguyen, M. M. Dobbin et al., "SIRT1 deacetylase protects against neurodegeneration in models for Alzheimer's disease and amyotrophic lateral sclerosis," The EMBO Journal, vol. 26, no. 13, pp. 3169-3179, 2007.

[51] W. Qin, T. Yang, L. Ho et al., "Neuronal SIRT1 activation as a novel mechanism underlying the prevention of alzheimer disease amyloid neuropathology by calorie restriction," The Journal of Biological Chemistry, vol. 281, no. 31, pp. 2174521754, 2006.

[52] D. J. Bonda, H. G. Lee, A. Camins et al., "The sirtuin pathway in ageing and Alzheimer disease: mechanistic and therapeutic 
considerations," The Lancet Neurology, vol. 10, no. 3, pp. 275279, 2011.

[53] P. Wareski, A. Vaarmann, V. Choubey et al., "PGC- $1 \alpha$ and PGC-1 $\beta$ regulate mitochondrial density in neurons," The Journal of Biological Chemistry, vol. 284, no. 32, pp. 2137921385, 2009.

[54] W. T. Johnson, L. A. K. Johnson, and H. C. Lukaski, "Serum superoxide dismutase 3 (extracellular superoxide dismutase) activity is a sensitive indicator of $\mathrm{Cu}$ status in rats," Journal of Nutritional Biochemistry, vol. 16, no. 11, pp. 682-692, 2005.

[55] J. M. McCord, "Iron- and manganese-containing superoxide dismutases: structure, distribution, and evolutionary relationships," Advances in Experimental Medicine and Biology, vol. 74, pp. 540-550, 1976.

[56] J. M. McCord and I. Fridovich, "Superoxide dismutase. An enzymic function for erythrocuprein (hemocuprein)," The Journal of Biological Chemistry, vol. 244, no. 22, pp. 60496055, 1969.

[57] S. L. Marklund, "Human copper-containing superoxide dismutase of high molecular weight," Proceedings of the National Academy of Sciences of the United States of America, vol. 79, no. 24 I, pp. 7634-7638, 1982.

[58] S. L. Marklund, "Extracellular superoxide dismutase in human tissues and human cell lines," Journal of Clinical Investigation, vol. 74, no. 4, pp. 1398-1403, 1984.

[59] F. Celsi, A. Ferri, A. Casciati et al., "Overexpression of superoxide dismutase 1 protects against $\beta$-amyloid peptide toxicity: effect of estrogen and copper chelators," Neurochemistry International, vol. 44, no. 1, pp. 25-33, 2004.

[60] C. Iadecola, F. Zhang, K. Niwa et al., "SOD1 rescues cerebral endothelial dysfunction in mice overexpressing amyloid precursor protein," Nature Neuroscience, vol. 2, no. 2, pp. 157$161,1999$.

[61] V. Blanchard, S. Moussaoui, C. Czech et al., "Time sequence of maturation of dystrophic neurites associated with $\mathrm{A} \beta$ deposits in APP/PS1 transgenic mice," Experimental Neurology, vol. 184, no. 1, pp. 247-263, 2003.

[62] J. S. Aucoin, P. Jiang, N. Aznavour et al., "Selective cholinergic denervation, independent from oxidative stress, in a mouse model of Alzheimer's disease," Neuroscience, vol. 132, no. 1, pp. 73-86, 2005.

[63] X. K. Tong, N. Nicolakakis, A. Kocharyan, and E. Hamel, "Vascular remodeling versus amyloid $\beta$-induced oxidative stress in the cerebrovascular dysfunctions associated with Alzheimer's disease," The Journal of Neuroscience, vol. 25, no. 48, pp. 11165-11174, 2005.

[64] R. A. Omar, Y. J. Chyan, A. C. Andorn, B. Poeggeler, N. K. Robakis, and M. A. Pappolla, "Increased expression but reduced activity of antioxidant enzymes in Alzheimer's disease," Journal of Alzheimer's Disease, vol. 1, no. 3, pp. 139$145,1999$.

[65] L. Esposito, J. Raber, L. Kekonius et al., "Reduction in mitochondrial superoxide dismutase modulates Alzheimer's disease-like pathology and accelerates the onset of behavioral changes in human amyloid precursor protein transgenic mice," The Journal of Neuroscience, vol. 26, no. 19, pp. 5167$5179,2006$.

[66] C. A. Massaad, T. M. Washington, R. G. Pautler, and E. Klann, "Overexpression of SOD-2 reduces hippocampal superoxide and prevents memory deficits in a mouse model of Alzheimer's disease," Proceedings of the National Academy of Sciences of the United States of America, vol. 106, no. 32, pp. 13576-13581, 2009.
[67] K. Fujita, Y. Nakabeppu, and M. Noda, "Therapeutic effects of hydrogen in animal models of Parkinson's disease," Parkinson's Disease, vol. 2011, Article ID 307875, 9 pages, 2011.

[68] M. Noda, K. Fujita, Lee C. H., and T. Yoshioka, "The principle and the potential approach to ROS-dependent cytotoxicity by non-pharmaceutical therapies: optimal use of medical gases with antioxidant properties," Current Pharmaceutical Design, vol. 17, no. 22, pp. 2253-2263, 2011.

[69] R. Von Burg, "Carbon monoxide," Journal of Applied Toxicology, vol. 19, no. 5, pp. 379-386, 1999.

[70] T. Sjostrand, "Early studies of CO production," Annals of the New York Academy of Sciences, vol. 174, no. 1, pp. 5-10, 1970.

[71] M. D. Maines, "The heme oxygenase system: update 2005," Antioxidants and Redox Signaling, vol. 7, no. 11-12, pp. 17611766, 2005.

[72] C. A. Piantadosi, "Carbon monoxide, reactive oxygen signaling, and oxidative stress," Free Radical Biology and Medicine, vol. 45, no. 5, pp. 562-569, 2008.

[73] D. Brancho, N. Tanaka, A. Jaeschke et al., "Mechanism of p38 MAP kinase activation in vivo," Genes and Development, vol. 17, no. 16, pp. 1969-1978, 2003.

[74] H. P. Kim, X. Wang, J. Zhang et al., " Heat shock protein70 mediates the cytoprotective effect of carbon monoxide: involvement of p38 $\beta$ MAPK and heat shock factor-1," Journal of Immunology, vol. 175, pp. 2622-2629, 2005.

[75] B. Wang, W. Cao, S. Biswal, and S. Dore, "Carbon monoxideactivated Nrf2 pathway leads to protection against permanent focal cerebral ischemia," Stroke, vol. 42, no. 9, pp. 2605-2610, 2011.

[76] S. R. Thom, D. Fisher, Y. A. Xu, K. Notarfrancesco, and H. Lschiropoulos, "Adaptive responses and apoptosis in endothelial cells exposed to carbon monoxide," Proceedings of the National Academy of Sciences of the United States of America, vol. 97, no. 3, pp. 1305-1310, 2000.

[77] S. Carballal, M. Trujillo, E. Cuevasanta et al., "Reactivity of hydrogen sulfide with peroxynitrite and other oxidants of biological interest," Free Radical Biology and Medicine, vol. 50, no. 1, pp. 196-205, 2011.

[78] M. Whiteman, J. S. Armstrong, S. H. Chu et al., "The novel neuromodulator hydrogen sulfide: an endogenous peroxynitrite "scavenger"?" Journal of Neurochemistry, vol. 90, no. 3, pp. 765-768, 2004.

[79] Z. Qingyou, D. Junbao, Z. Weijin, Y. Hui, T. Chaoshu, and Z. Chunyu, "Impact of hydrogen sulfide on carbon monoxide/heme oxygenase pathway in the pathogenesis of hypoxic pulmonary hypertension," Biochemical and Biophysical Research Communications, vol. 317, no. 1, pp. 30-37, 2004.

[80] C. Szabo, "Hydrogen sulphide and its therapeutic potential," Nature Reviews Drug Discovery, vol. 6, no. 11, pp. 917-935, 2007.

[81] I. Ohsawa, M. Ishikawa, K. Takahashi et al., "Hydrogen acts as a therapeutic antioxidant by selectively reducing cytotoxic oxygen radicals," Nature Medicine, vol. 13, no. 6, pp. 688-694, 2007.

[82] K. Nagata, N. Nakashima-Kamimura, T. Mikami, I. Ohsawa, and S. Ohta, "Consumption of molecular hydrogen prevents the stress-induced impairments in hippocampus-dependent learning tasks during chronic physical restraint in mice," Neuropsychopharmacology, vol. 34, no. 2, pp. 501-508, 2009.

[83] Y. Gu, C. S. Huang, T. Inoue et al., "Drinking hydrogen water ameliorated cognitive impairment in senescence-accelerated mice," Journal of Clinical Biochemistry and Nutrition, vol. 46, no. 3, pp. 269-276, 2010. 
[84] K. I. Fukuda, S. Asoh, M. Ishikawa, Y. Yamamoto, I. Ohsawa, and S. Ohta, "Inhalation of hydrogen gas suppresses hepatic injury caused by ischemia/reperfusion through reducing oxidative stress," Biochemical and Biophysical Research Communications, vol. 361, no. 3, pp. 670-674, 2007.

[85] B. M. Buchholz, D. J. Kaczorowski, R. Sugimoto et al., "Hydrogen inhalation ameliorates oxidative stress in transplantation induced intestinal graft injury," American Journal of Transplantation, vol. 8, no. 10, pp. 2015-2024, 2008.

[86] A. Nakao, D. J. Kaczorowski, Y. Wang et al., "Amelioration of rat cardiac cold ischemia/reperfusion injury with inhaled hydrogen or carbon monoxide, or both," Journal of Heart and Lung Transplantation, vol. 29, no. 5, pp. 544-553, 2010.

[87] K. Hayashida, M. Sano, I. Ohsawa et al., "Inhalation of hydrogen gas reduces infarct size in the rat model of myocardial ischemia-reperfusion injury," Biochemical and Biophysical Research Communications, vol. 373, no. 1, pp. 30-35, 2008.

[88] I. Ohsawa, K. Nishimaki, K. Yamagata, M. Ishikawa, and S. Ohta, "Consumption of hydrogen water prevents atherosclerosis in apolipoprotein E knockout mice," Biochemical and Biophysical Research Communications, vol. 377, no. 4, pp. 1195-1198, 2008.

[89] K. Fujita, T. Seike, N. Yutsudo et al., "Hydrogen in drinking water reduces dopaminergic neuronal loss in the 1-methyl4-phenyl-1,2,3,6-tetrahydropyridine mouse model of Parkinson's disease," PLoS ONE, vol. 4, no. 9, Article ID e7247, 2009.

[90] Y. Fu, M. Ito, Y. Fujita et al., "Molecular hydrogen is protective against 6-hydroxydopamine-induced nigrostriatal degeneration in a rat model of Parkinson's disease," Neuroscience Letters, vol. 453, no. 2, pp. 81-85, 2009.

[91] F. Fornai, O. M. Schlüter, P. Lenzi et al., "Parkinson-like syndrome induced by continuous MPTP infusion: convergent roles of the ubiquitin-proteasome system and $\alpha$-synuclein," Proceedings of the National Academy of Sciences of the United States of America, vol. 102, no. 9, pp. 3413-3418, 2005.

[92] K. I. Setsukinai, Y. Urano, K. Kakinuma, H. J. Majima, and T. Nagano, "Development of novel fluorescence probes that can reliably detect reactive oxygen species and distinguish specific species," The Journal of Biological Chemistry, vol. 278, no. 5, pp. 3170-3175, 2003.

[93] L. P. Liang, J. Huang, R. Fulton, B. J. Day, and M. Patel, “An orally active catalytic metalloporphyrin protects against 1methyl-4-phenyl-1,2,3,6-tetrahydropyridine neurotoxicity in vivo," The Journal of Neuroscience, vol. 27, no. 16, pp. 43264333, 2007.

[94] Y. Sato, S. Kajiyama, A. Amano et al., "Hydrogen-rich pure water prevents superoxide formation in brain slices of vitamin C-depleted SMP30/GNL knockout mice," Biochemical and Biophysical Research Communications, vol. 375, no. 3, pp. 346350, 2008.

[95] A. Nakao, Y. Toyoda, P. Sharma, M. Evans, and N. Guthrie, "Effectiveness of hydrogen rich water on antioxidant status of subjects with potential metabolic syndrome-an open label pilot study," Journal of Clinical Biochemistry and Nutrition, vol. 46, no. 2, pp. 140-149, 2010. 


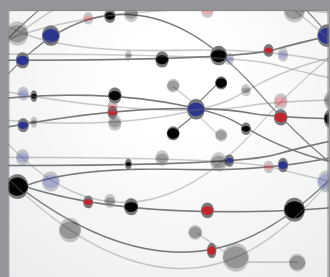

The Scientific World Journal
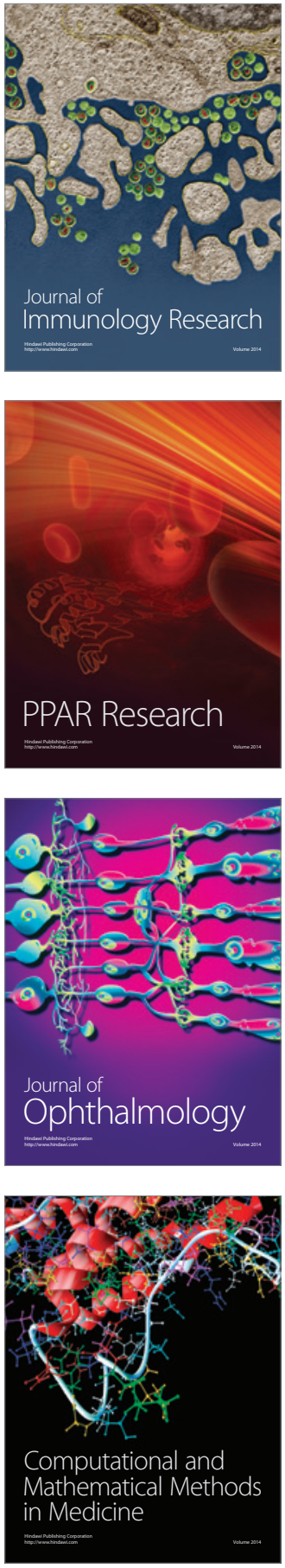

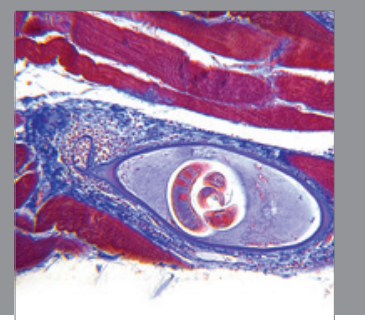

Gastroenterology

Research and Practice
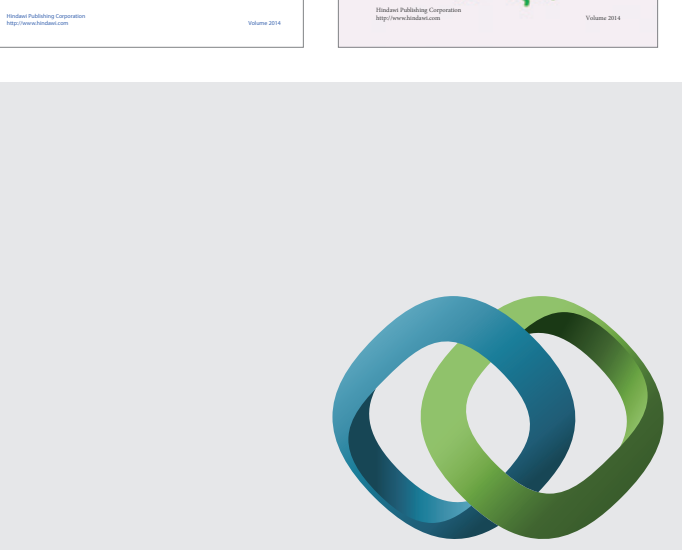

\section{Hindawi}

Submit your manuscripts at

http://www.hindawi.com
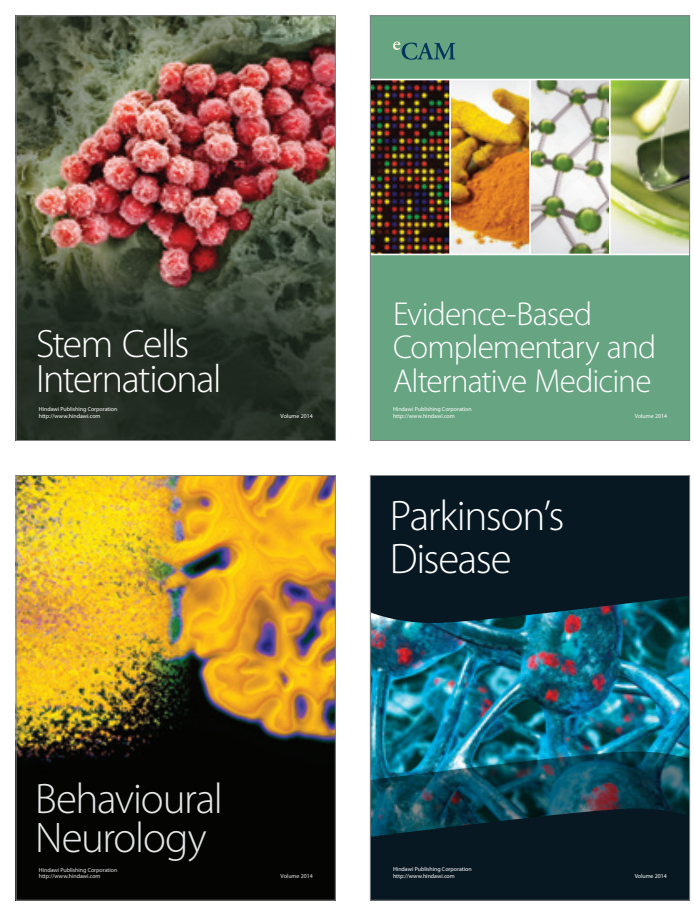

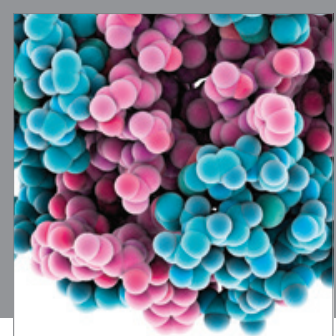

Journal of
Diabetes Research

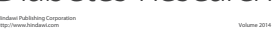

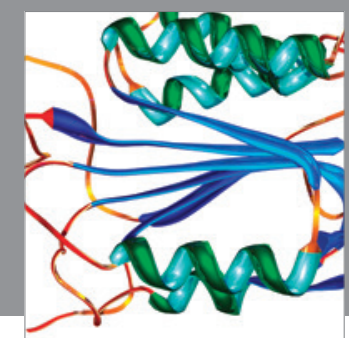

Disease Markers
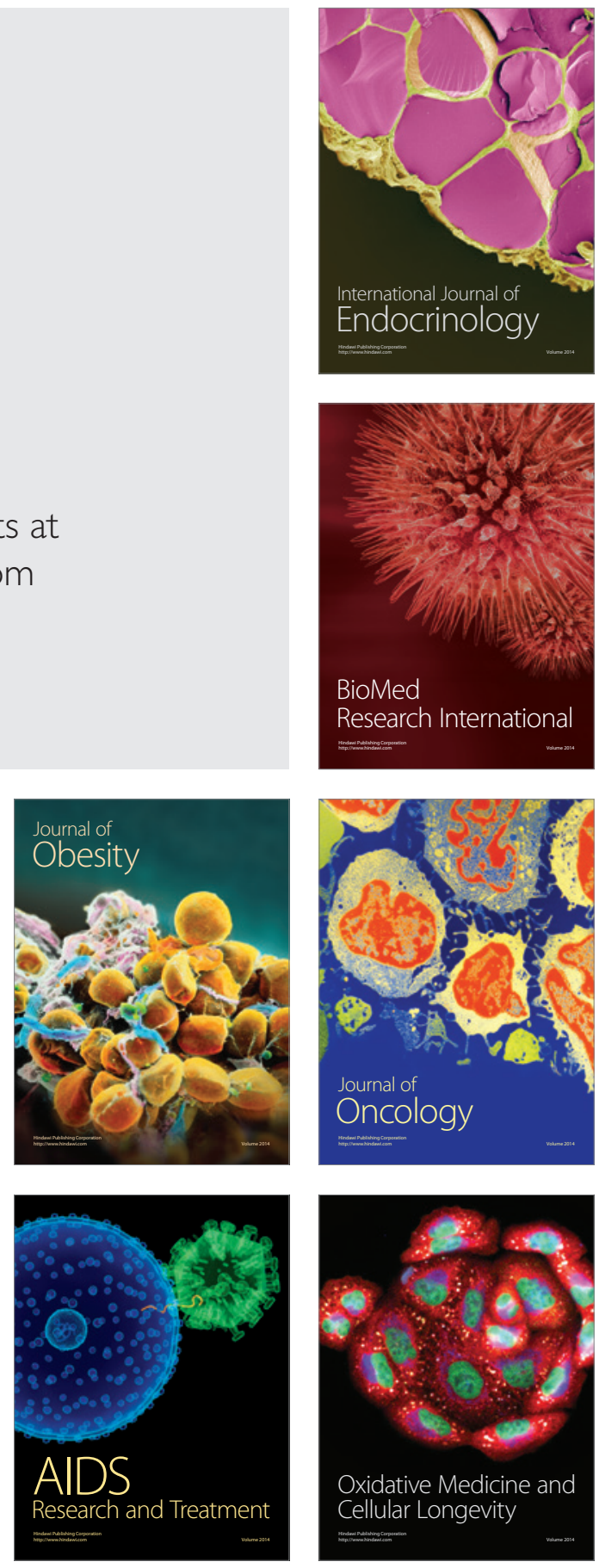